\title{
Can Balance Function Tests Predict Disability in Older Adults with Peripheral Vestibular Hypofunction?
}

\author{
Amie F. Jasper ${ }^{\circledR 1}$, Mary Blackinton ${ }^{(2)}$, Joann Gallichio ${ }^{\circledR 3}$, Anne K. Galgon ${ }^{(1)}$ \\ ${ }^{1}$ University of St. Augustine for Health Sciences, College of Rehabilitative Sciences, St. Augustine, FL, USA \\ ${ }^{2}$ enTandem DPT, Rehab Essentials, Columbia Falls, MT, USA \\ ${ }^{3}$ Nova Southeastern University, College of Health Care Sciences, Ft. Lauderdale, FL, USA \\ ${ }^{4}$ University of the Sciences, Department of Physical Therapy, Philadelphia, PA, USA \\ *Address all correspondence to Amie M. Jasper at: amjasper@usa.edu
}

To cite this article: Jasper, A.F., Blackinton, M., Gallichio, J., Galgon, A.K. (2022). Can balance function tests predict disability in older adults with peripheral vestibular hypofunction? Philippine Journal of Physical Therapy, 1(1), 16-24. https://doi.org/10.46409/002.CRLW5593

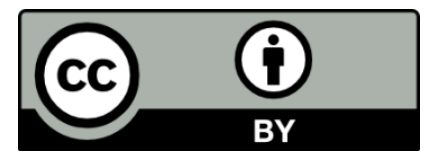

This article is licensed under the Creative Commons Attribution 4.0 International License (CC-BY 4.0). You are free to copy and distribute the work under the following terms: You must give appropriate credit and include a link to the original work. This cover page or a standard citation including the DOI link will meet this term. You must also include the link to the CC-BY license.

\begin{abstract}
Introduction: The Dizziness Handicap Inventory (DHI) is a 25-item self-assessment questionnaire used to evaluate perceived disability from dizziness. The predictive validity of Timed Up and Go (TUG), Dynamic Gait Index (DGI), and modified Clinical Test of Sensory Integration of Balance (mCTSIB) on disability as shown by DHI has not been established specific to older adults with peripheral vestibular hypofunction. The purpose of this study is to investigate if the TUG, DGI, and mCTSIB are significant and strong predictors of the disability from dizziness as represented by the DHI scores in older adults with peripheral vestibular hypofunction.

Method: A Correlational, Retrospective Design was used to investigate the predictors of the DHI score. Data was collected retrospectively on the 17 patients in the main study 'The Impact of Prescribed Walking on Dizziness in Seniors with Vestibular Hypofunction: A Pilot Randomized Controlled Trial' and from physical therapy medical charts ( $\mathrm{n}=24)$ from June 2015 to June 2018. Only medical charts of those 65 years and older who underwent vestibular physical therapy and contain all four outcome measures (DHI, DGI, mCTSIB, and TUG) were included in the study. Dizziness related to central nervous disorder were excluded.

Results: There was a significant fair inverse correlation between DHI and mCTSIB $(r=-0.381, p=0.01)$ and DGI $(r=-0.322, p=$ $0.01)$, and a fair correlation with TUG $(r=0.396, p=0.03)$. The mCTSIB, TUG, and DGI accounted for $10 \%$ of the variance in the DHI, however, this predictive relationship was not found to be significant.

Discussion: In this limited sample size, the TUG, DGI, and mCTSIB tests are not significant and strong predictors of dizziness-related disability as represented by the DHI scores in older adults with peripheral vestibular dysfunction. This study should be replicated as a large-scale prospective study with stratification of severity of dizziness to improve the generalizability of findings.
\end{abstract}

Keywords: Older Adults, Disability, Dizziness, Dizziness Handicap Inventory, Peripheral Vestibular Hypofunction 


\section{Introduction}

Dizziness is a common complaint reported by older adults (Maarsingh et al., 2014). Disorders of the vestibular system are responsible for 40 to $50 \%$ of dizziness, and peripheral vestibular disorders in older adults are common (Alrwaily \& Whitney, 2011). Dizziness can result from vestibular dysfunction or from non-vestibular causes such as sensory loss, psychiatric and cardiovascular disorders, postural hypotension, and adverse drug effects (Bovo et al., 2014). Most community-dwelling older adults who are suffering from dizziness have more than one underlying cause of dizziness (Fernández et al., 2015).

Older adults with dizziness reported to have great physical and psychosocial burden (Hsu et al., 2005). The Dizziness Handicap Inventory (DHI) is a 25-item self-assessment questionnaire that measures the impact of dizziness on the physical, functional and emotional domains of disability (Jacobson \& Newman, 1990). Higher score indicates more perceived disability from dizziness. The Vestibular Evidence Database to Guide Effectiveness (VEDGE) task force of the Academy of Neurologic Physical Therapy (ANPT) recommends the DHI for evaluation of the level of participation on the International Classification of Functioning, Disability and Health (ICF) model (ANPT, 2013). Participation refers to the ability to participate in life's roles such as homemaker or worker.

Patients with dizziness have activity limitations and participation restrictions due to actual and perceived balance problems (Mueller et al., 2014). According to Gananca et al. (2004), selfreported (subjective) DHI has a moderate correlation with objective tests for balance and functional performance. In older adults with complaints of dizziness related to vestibular dysfunction, the DHI was significantly higher among fallers than nonfallers (Gananca et al., 2004). Moreover, individuals with impaired postural control as represented by low scores (less than 30 seconds in one or more of the four conditions) in modified Clinical Test of Sensory Integration of Balance (mCTSIB) reported greater severity in the DHI scores (Whitney \& Wrisley, 2004). Outcome measures that quantify functional mobility, walking ability, and fall risk, such as the Timed Up and Go (TUG) test and Dynamic Gait Index (DGI) were cited as predictors of the DHI scores (Jacobson et al., 1991; Vereeck et al., 2007). The TUG, together with balance tests and posturography explained $78 \%$ of the variance in DHI in patients with bilateral vestibular hypofunction but not for unilateral vestibular hypofunction, while in another study, the DGI explained $42 \%$ of the variance in DHI scores (Jacobson et al., 1991; Vereeck et al., 2007).

Age had a fair relationship with the DHI total score, however, previous studies on the predictive validity of the DHI were performed on samples with heterogenous age groups, combining both adults and older adults (age range 20.3 - 92.2 years) within a single study (Jacobson et al., 1991; Vereeck et al., 2007). It was unknown whether impairments of body function and activity limitations predicted dizziness-related disability in older adults. Understanding the usefulness of TUG, DGI, and mCTSIB as potential determinants of perceived disability in older adults with a peripheral vestibular disorder could guide physical therapists to address the participation restrictions associated with dizziness. The purpose of this study is to investigate if the TUG, DGI, and mCTSIB are significant and strong predictors of dizziness-related disability as measured by the Dizziness Handicap Inventory in older adults with peripheral vestibular hypofunction.

\section{Method}

\section{Study Design}

A correlational, retrospective design was used to investigate the predictors of the DHI approved by the Institutional Review Boards of Advent Health, Advent Health University, and Nova Southeastern University. This study is registered under Clinicaltrials.gov. Due to the study's retrospective design, obtaining informed consent was not applicable.

Data were extracted from 17 patients included in the main study 'The Impact of Prescribed Walking on Dizziness in Seniors with Vestibular Hypofunction: A Pilot Randomized Controlled Trial'. To supplement the data, a retrospective chart review was conducted on the medical charts of patients of Advent Health Sports Medicine and Rehabilitation 65 years old or older $(n=24)$ who had vestibular physical therapy initial evaluation and first physical therapy visit from June 2015 to June 2018.

\section{Subjects}

Medical charts of patients who were 65 years and older who underwent vestibular physical therapy and contain all four outcome measures (DHI, DGI, mCTSIB and TUG) were included in the study through convenience sampling. The four outcome measures were administered by different physical therapists. Dizziness related to central nervous system disorder, such as stroke, head injuries, multiple sclerosis or parkinson's disease were excluded.

\section{Description of Outcome Measures}

\section{DHI}

The DHI is a self-report outcome measure that has 25 questions on the functional, emotional, and physical impact of dizziness. An ordinal scale is used to answer the questions with zero for no, two for sometimes, and four for yes. The total score can range from 0 to 100 . The severity of dizziness can be interpreted as mild for scores between 0-30, moderate for 31-60, and severe for 61-100 
(Jacobson \& Newman, 1990). An example of a question from the DHI is "Does your dizziness interfere with your job or household responsibilities?". The DHI has been found to have an excellent negative correlation $(r=-0.64)$ with the Activity Specific Balance Confidence Scale (ABC) in the older adults and patients with vestibular dysfunction (Whitney et al., 1999). Furthermore, it has excellent correlation with SF-36 $(r=0.53-0.72)$ (Fielder et al., 1996) and moderate statistically significant negative correlation with Sensory Organization Test conditions $2(\mathrm{r}=-0.39), 4(\mathrm{r}=-$ $0.36), 5(\mathrm{r}=-0.42)$ and $6(\mathrm{r}=-0.35)$ in individuals with vestibular disorder (Jacobson et al., 1991).

\section{$T U G$}

The TUG is a test of functional mobility and gait, requiring a person to come to standing, walk three meters, turn around and return to the chair. It is utilized to assess fall risk (Shumway-Cook et al., 2000). Among the population studied for the TUG are the frail elderly and vestibular disorders (Podsiadlo \& Richardson, 1991). The cut-off scores that indicate risk for falls are greater than 13.5 seconds for community-dwelling older adults (Shumway-Cook et al., 2000) and greater than 11.1 seconds for vestibular disorders (Whitney et al., 2004). Interrater reliability was excellent for hospitalized and community-dwelling older individuals $(\mathrm{ICC}=0.93$ ) (Kristensen et al., 2019). Podsiadlo and Richardson (1991) found that in the elderly adults, the TUG has an excellent correlation with Berg Balance $(r=-0.81)$, gait speed $(\mathrm{r}=-0.61)$, and Barthel Index of ADL $(\mathrm{r}=-0.78)$. It has $80 \%$ sensitivity and $56 \%$ specificity in falls prediction for vestibulopathic older individuals (Whitney et al., 2004).

\section{DGI}

The DGI assesses the ability to maintain balance while walking in the presence of external demands such as walking with head turns, stepping over an obstacle, turning, and stairclimbing. Scores are 3 for no gait dysfunction, 2 for minimal impairment, 1 for moderate impairment and 0 for severe impairment with the total score ranging from 0 to 24 (Wrisley et al., 2003). A cut-off score of less than 19 is indicative of increased fall risks in communitydwelling older adult (Shumway-Cook et al., 1997). According to Jonsson et al. (2011), DGI has an excellent intrarater $(\mathrm{ICC}=0.89)$ and interrater $(\mathrm{ICC}=0.82$ ) reliability for community-dwelling older adults with baseline impairment. For the vestibular population, it has an adequate inter-rater reliability $(\mathrm{k}=0.64)$ (Wrisley et al., 2003). Shumway-Cook et al. (1997) found the DGI to have excellent correlation with Balance Self-Perceptions test ( $\mathrm{r}$ $=0.76)$ and Berg Balance Test $(r=0.67)$ and adequate correlation with assistive devices history $(r=-0.44)$ and history of imbalance $(\mathrm{r}=-0.46)$ in the geriatric population. It has an excellent concurrent validity with the Berg Balance Scale $(r=0.71)$ in the vestibular population (Whitney et al., 2003).

\section{MCTSIB}

The mCTSIB checks the ability of the patient to utilize information from somatosensory, visual, and vestibular systems effectively for postural control. This test is the short version of the original CTSIB which uses an altered visual input in conditions 3 and 6 (Horn et al., 2015). The patient is timed for 30 seconds on each condition which are standing on firm surface eyes open, standing on firm surface eyes closed, standing on compliant surface eyes open, and standing on compliant surface eyes closed. The average score of three trials is obtained with the highest possible score of 120 seconds. Failure to maintain the balance for 30 seconds in condition 2 indicates that the patient is visually dependent (O'Sullivan et al., 2019). The patient is not utilizing the visual and vestibular systems well if the patient is not able to maintain the balance for conditions 3 and 4 (O'Sullivan et al., 2019). To date, there is no established cut-off scores for fall risk prediction for mCTSIB. Significant but small correlations were found between $\mathrm{mCTSIB}$ scores and SOT condition 2, firm surface with eyes closed $(r=0.48)$; condition 4 , sway referenced surface, eyes open $(r=0.30)$; and condition 5 , sway-referenced surface with eyes closed $(\mathrm{r}=0.51)$ in patients with complaints of dizziness or imbalance (Wrisley \& Whitney, 2004). MCTSIB, a less costly alternative to computerized analysis of balance has been found to have a good agreement (kappa values 0.53 - 0.81) between two testers (Loughran et al., 2005).

\section{Data Extraction}

Age, gender, treatment diagnosis, and outcome scores for DHI, DGI, mCTSIB, and TUG were extracted from the medical charts and recorded on the data collection spreadsheet. There was no link between the extracted data and medical record.

\section{Statistical Analysis}

Based on the DHI total scores, the patients were grouped into mild (0-30), moderate (31-60), and severe (61-100) (Whitney et al., 2004) to obtain a better picture between the dependent variable DHI and the independent variables: mCTSIB, TUG, and DGI (Vereeck et al., 2007). Kruskal-Wallis test was then calculated to compare the age, mCTSIB, TUG, and DGI scores of the groups. A post-hoc analysis was done using the Bonferroni correction for multiple tests.

To determine the relationship between DHI and mCTSIB, TUG and DGI, the Spearman Rho correlation coefficient was used. As a general guideline, the strength of relationship was based on the proximity of the values of $r$ to 1 (Portney \& Watkins, 2009).

To determine the predictors of DHI, a multiple linear regression analysis was conducted on the three independent variables which are mCTSIB, TUG, and DGI. The assumptions for a multiple linear regression analysis were first tested. The results of the Durbin-Watson statistic showed no correlation between residuals and an independence of observations. 


\section{Results}

Data on age, gender, treatment diagnosis, DHI, DGI, mCTSIB, and TUG were extracted from medical charts of 41 patients through convenience sampling: 17 from an ongoing study and 24 from a retrospective chart review. The mean age was 77.59 years old and the mean DHI score was 31.56. There were 27 females and 14 males. The medical diagnosis combined with the clinical examination findings of the physical therapists were consistent with peripheral vestibular hypofunction in all patients. The mean, standard deviation, and range for age, DHI, mCTSIB, TUG, and DHI were shown in Table 1.

There were significant differences between the mild, moderate, and severe DHI groups on mCTSIB $(\mathrm{p}=0.04)$, TUG $(\mathrm{P}=0.03)$ and DGI $(\mathrm{p}=0.04)$ as shown in Table 2. After pairwise comparisons, significant difference was found only in the TUG scores $(p=0.049)$ between mild and moderate groups. There were no between group differences in the other outcomes.

None of the obtained correlations were above $r=0.7$ or a strong linear relationship as shown in Table 3. There were significant fair inverse correlations between DHI and mCTSIB $(r=-0.381, p=$ $0.01)$ and DGI $(r=-0.322, p=0.01)$, and a fair correlation with TUG $(r=0.396, p=0.03)$.

There was an independence of observations based on the DurbinWatson statistics value of 2.639. The assumptions of homoscedasticity, normality, and multicollinearity were met. There were no outliers in the data.

The result of the multiple regression analysis showed that the independent variables account for $10.5 \%$ of the variance in the dependent variable, however the predictive relationship did not reach significance $(p=0.246)$ as shown in Table 4 . None of the variables which are mCTSIB, TUG, and DGI were significant predictors of the DHI total scores.

\section{Discussion}

When the subjects were divided into mild, moderate, and severe groups based on their DHI scores, significant differences were observed on mCTSIB $(p=0.04)$, TUG $(p=0.03)$, and DGI $(p=$ 0.04). This result was similar to the findings of the retrospective study of Vereeck et al. (2007) on patients with dizziness in the balance clinic. After pairwise comparisons, our study found a significant difference only in TUG $(\mathrm{p}=0.049)$ between the mild and moderate groups. On the contrary, Vereeck et al. (2007) derived significant differences between the three groups on all balance tests. The differences in findings between the two studies could be explained by age, the number of total and between-group samples, and etiology. The mean age in Vereeck et al. (2007) study was lower (mean $=53.9$ years, range $=23-87$ years) compared to our study (mean age $=77.59$ years, range $=66-94$ years). Also, they had cases of both vestibular and nonvestibular disorders, and a few cases of central origin (Vereeck et al., 2007). Conversely, our sample represented only the geriatric population which narrowed down our target patients. Furthermore, our sample size was lower since we only included those with peripheral vestibular hypofunction and excluded dizziness related to central nervous system disorder. Our severe group was composed of four patients only, which may have limited its potential to reveal significant difference between the mild-severe and moderate-severe groups.

Prior research showed fair relationship between DHI and static balance measures, and moderate relationship between DHI and walking tests such as TUG and DGI (Vereeck et al., 2007). GillBody et al. (2000) found a fair relationship between DHI and Sensory Organization Test conditions 1 and 3 and feet together with eyes closed, and moderate relationship between DHI and TUG. Our study has seen a similar result for mCTSIB $(r=-0.381)$ but not for TUG $(r=-0.396)$ and DGI $(r=-0.322)$ in older adults with peripheral vestibular hypofunction. Both walking tests have fair relationships with the DHI. It is possible that the gait instability experienced by the older adults could be multifactorial in nature, rather than being related mainly to dizziness. Compared to the subjects (age range 20 to 92 years old) in the studies of GillBody et al. (2000) and Vereeck et al. (2007), age-related decline in balance and walking performances among our participants could also contribute to this discrepancy in results.

In our study, we found that mCTSIB, TUG, and DGI are not strong and significant predictors of the disability from dizziness in older adults with peripheral vestibular hypofunction. This result is in contrasts with two previous studies that utilized heterogenous age groups of adults and older adults. In these studies, the DGI explained for $42 \%$ of the variance of DHI for patients visiting the balance clinic between ages 23 to 87 years old (mean 53.9, $\mathrm{n}=$ 214) (Gill-Body et al., 2000) and the TUG combined with two balance measures accounted for $78 \%$ of the variance of DHI in patients with bilateral vestibular hypofunction between ages 20 to 92 years old (mean 62.5, $\mathrm{n}=44$ ) (Vereeck et al., 2007). Thus, predictors other than balance and walking tests should to be explored in older adults with peripheral vestibular hypofunction. In fact, in the final logistic model of Dros et al. (2012) on older adults with dizziness from vestibular and non-vestibular origin, chronic dizziness greater than 6 months, standing still, trouble with walking, polypharmacy, absence of diabetes, anxiety, or depressive disorder, impaired functional mobility, and TUG were variables that are most predictive of disability from dizziness in older adults.

The impact of dizziness in older adults is most likely to be multidimensional. The DHI is an outcome measure that quantifies the physical, psychosocial, and emotional effects of dizziness. Our study suggests that clinicians may combine balance and functional tests with factors in the clinical history to explore the predictors of disability from dizziness. 


\section{Table 1}

Subject Characteristic $(n=41)$

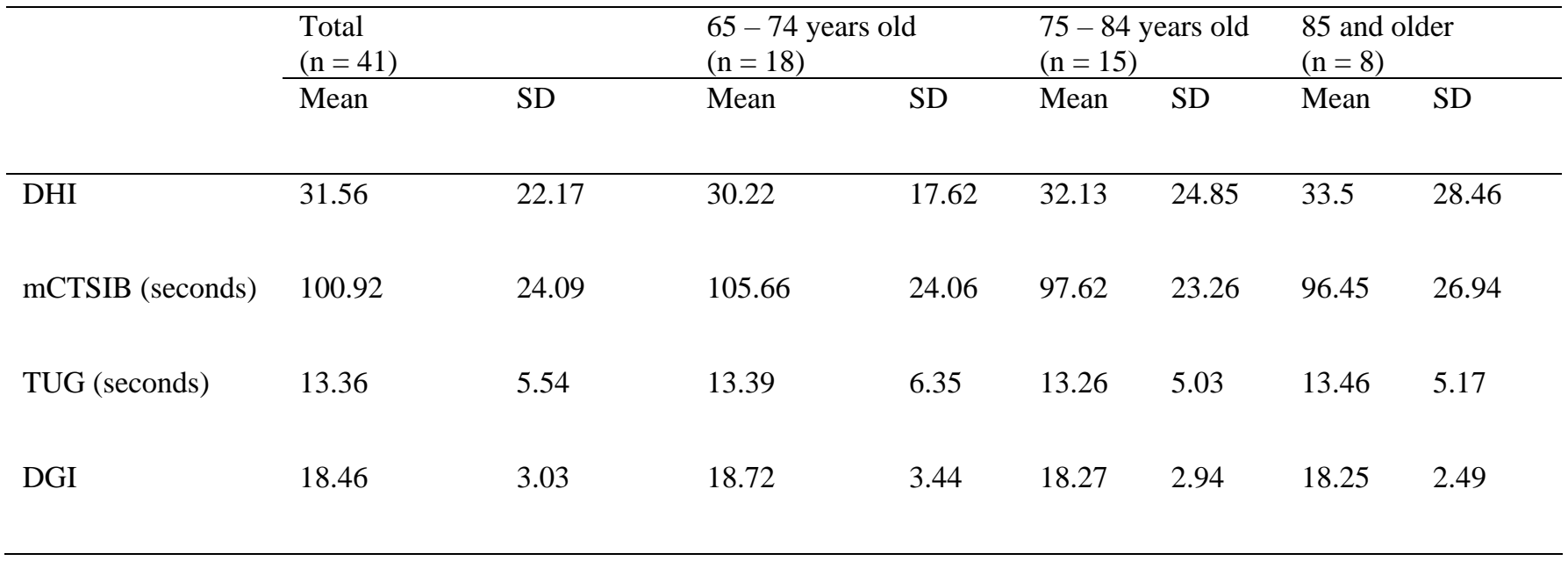

Note. DHI, Dizziness Handicap Inventory; mCTSIB, Modified Clinical Test for Sensory Integration for Balance; TUG, Timed Up and Go; DGI, Dynamic Gait Index; SD, Standard Deviation

\section{Table 2}

Means and Standard Deviations of mCTSIB, TUG and DGI Results for Each of the Three DHI Groups ( $n=41)$

\begin{tabular}{|c|c|c|c|c|c|c|c|}
\hline \multirow[t]{2}{*}{ Variables } & \multicolumn{2}{|c|}{$\begin{array}{l}\text { Mild DHI 0-30 } \\
(\mathrm{n}=24)\end{array}$} & \multicolumn{2}{|c|}{$\begin{array}{l}\text { Moderate DHI 31-60 } \\
(\mathrm{n}=13)\end{array}$} & \multicolumn{2}{|c|}{$\begin{array}{l}\text { Severe DHI 61-100 } \\
(\mathrm{n}=4)\end{array}$} & \multirow{2}{*}{ P-value } \\
\hline & Mean & $\mathrm{SD}$ & Mean & $\mathrm{SD}$ & Mean & $\mathrm{SD}$ & \\
\hline Age & 76.92 & 7.73 & 77.85 & 8.31 & 80.75 & 7.59 & 0.69 \\
\hline mCTSIB & 108.89 & 15.73 & 86.42 & 32.51 & 100.25 & 14.00 & 0.04 \\
\hline TUG & 11.82 & 5.04 & 16.08 & 6.05 & 13.75 & 4.11 & 0.03 \\
\hline DGI & 19.50 & 2.77 & 17.08 & 3.17 & 16.75 & 1.71 & 0.04 \\
\hline
\end{tabular}

Note. DHI, Dizziness Handicap Inventory; mCTSIB, Modified Clinical Test for Sensory Integration for Balance; TUG, Timed Up and Go; DGI, Dynamic Gait Index; SD, Standard Deviation 
Table 3

Correlations Between the DHI Total Scores, $m$ CTSIB, TUG, and DGI $(n=41)$

\begin{tabular}{llll}
\hline & mCTSIB & TUG & DGI \\
\hline DHI total scores & -0.381 & 0.396 & -0.322 \\
P-value & 0.01 & 0.01 & 0.04 \\
& & & \\
\hline
\end{tabular}

Note: TUG, Timed Up and Go; DGI, Dynamic Gait Index; DHI, Dizziness Handicap Inventory; mCTSIB, Modified Clinical Test for Sensory Integration for Balance

\section{Table 4}

Multiple Linear Regression with DHI as the Dependent Variable and mCTSIB, TUG and DGI as the Independent Variables

\begin{tabular}{|c|c|c|c|c|c|c|c|c|c|c|}
\hline \multirow[b]{2}{*}{ Model $^{\mathrm{b}}$} & \multirow[b]{2}{*}{$\mathrm{R}$} & \multirow[b]{2}{*}{$\begin{array}{l}\mathrm{R} \\
\text { Square }\end{array}$} & \multirow[b]{2}{*}{$\begin{array}{l}\text { Adjusted } \\
\text { R Square }\end{array}$} & \multirow[b]{2}{*}{$\begin{array}{l}\text { Std. Error } \\
\text { of the } \\
\text { Estimate }\end{array}$} & \multicolumn{5}{|c|}{ Change Statistics } & \multirow[b]{2}{*}{$\begin{array}{l}\text { Durbin- } \\
\text { Watson }\end{array}$} \\
\hline & & & & & $\begin{array}{l}\mathrm{R} \\
\text { Square } \\
\text { Change }\end{array}$ & $\begin{array}{l}\text { F } \\
\text { Change }\end{array}$ & df1 & df2 & $\begin{array}{l}\text { Sig. F. } \\
\text { Change }\end{array}$ & \\
\hline 1 & $0.324^{\mathrm{a}}$ & 0.105 & 0.032 & 21.80979 & 0.105 & 1.444 & 3 & 37 & 0.246 & 2.639 \\
\hline
\end{tabular}

Note. Std, Standard; df, Degrees of Freedom; Sig, Significant; DGI, Dynamic Gait Index; mCTSIB, Modified Clinical Test for Sensory Integration for Balance; TUG, Timed Up and Go; DHI, Dizziness Handicap Inventory

${ }^{\text {a }}$ Predictors: (Constant), DGI, MCTSIB, TUG

${ }^{\mathrm{b}}$ Dependent Variable: DHI 
The patients were recruited through convenience sampling. As a result, there was low number of participants in the severe category which most likely reflect the outpatient setting. Therefore, the generalization of our results is limited among the older adults with mild to moderate dizziness from peripheral vestibular hypofunction.

Lastly, it was no longer possible to address the threats to internal validity that arise from the administration of tests due to the retrospective nature of the study. Only the overall scores of DHI were extracted from the medical charts. The DHI questionnaires were not available for view to obtain the scores on physical, functional and emotional domains of DHI. It would be interesting to know the predictive potential of mCTSIB, TUG, and DGI on each DHI domain.

For future research, we recommend a stratification sampling method based on the DHI severity for equal representation of subjects with mild, moderate, and severe disability from dizziness. A prospective multicenter research design with a larger sample size would allow researchers to include variables from the clinical history such as onset of dizziness, polypharmacy, presence of diabetes, depression, and anxiety in the regression model. Statistical analysis can be performed on the physical, functional and domains of DHI to check the correlation and predictive potential of $\mathrm{mCTSIB}, \mathrm{TUG}$, and DGI on each domain.

\section{Conclusion}

In this retrospective study, the TUG, DGI, and mCTSIB were not strong and significant predictors of dizziness-related disability for older adults with peripheral vestibular dysfunction. Clinicians should be cautious in predicting disability from dizziness based solely on the balance and walking outcomes. Other factors in the clinical history may need to be considered such as onset of dizziness, polypharmacy, presence of diabetes, depression and anxiety. We also suggest future studies to have a large-scale prospective research design and stratification sampling based on the DHI score for equal representation of the severity of the disability from dizziness.

\section{Conflict of interest statement}

The authors declare that they have no conflict of interest with regard to the research conducted in this paper.

\section{Acknowledgement}

The authors would like to thank the research sponsor, Advent Health (former Florida Hospital), grant sponsor Advent Health University (former Adventist University), and research team: Dr. Roy Lukman, Dr. Leana Araujo, Dr. Trevor Hicks, Elizabeth
Rosales, Myrna Santiago, Susan Thome-Barrett and Grace Bacani.

\section{References}

Alrwaily, M., \& Whitney, S. L. (2011). Vestibular rehabilitation of older adults with dizziness. Otolaryngolic Clinics of North America, 44(2), 473-x. https://doi.org/10.1016/j.otc.2011.01.015

ANPT (2013). VEDGE Measures Recommended for Research. http://www.neuropt.org/docs/default-source/vestibularedge/vedge-measures-recommended-forresearch.pdf?sfvrsn $=2$

Bovo, R., Faccioli, C., \& Martini, A. (2014). Dizziness in the elderly. Hearing, Balance and Communication, 12(2), 54-65. https://doi.org/10.3109/21695717.2013.871793

Dros, J., Maarsingh, O. R., Beem, L., van der Horst, H. E., ter Riet, G., Schellevis, F. G., \& van Weert, H. C. (2012). Functional prognosis of dizziness in older adults in primary care: a prospective cohort study. Journal of the American Geriatrics Society, 60(12), 2263-2269. https://doi.org/10.1111/jgs.12031

Fielder, H., Denholm, S. W., Lyons, R. A., \& Fielder, C. P. (1996). Measurement of health status in patients with vertigo. Clinical Otolaryngology and Allied Sciences, 21(2), 124126. https://doi.org/10.1111/j.1365-2273.1996.tb01314.x

Fernández, L., Breinbauer, H. A., \& Delano, P. H. (2015). Vertigo and dizziness in the elderly. Frontiers in Neurology, 6, 144. https://doi.org/10.3389/fneur.2015.00144

Gananca, F. F., Cavalli, S. S., Gazzola, J., \& Perracini, M. R. (2004). Are falls a good predictor of health-related quality of life in dizzy elderly?. Otolaryngology - Head and Neck Surgery, 131(2), 158. https://doi.org/10.1016/j.otohns.2004.06.269

Gill-Body, K. M., Beninato, M., \& Krebs, D. E. (2000). Relationship among balance impairments, functional performance, and disability in people with peripheral vestibular hypofunction. Physical Therapy, 80(8), 748-758. https://doi.org/10.1093/ptj/80.8.748

Horn, L. B., Rice, T., Stoskus, J. L., Lambert, K. H., Dannenbaum, E., \& Scherer, M. R. (2015). Measurement characteristics and clinical utility of the clinical test of sensory interaction on balance (CTSIB) and modified CTSIB in individuals with vestibular dysfunction. Archives of 
Physical Medicine and Rehabilitation, 96(9), 1747-1748. https://doi.org/10.1016/j.apmr.2015.04.003

Hsu, L. C., Hu, H. H., Wong, W. J., Wang, S. J., Luk, Y. O., \& Chern, C. M. (2005). Quality of life in elderly patients with dizziness: analysis of the Short-Form Health Survey in 197 patients. Acta Oto-laryngologica, 125(1), 55-59. https://doi.org/10.1080/00016480410017512

Jacobson, G. P., \& Newman, C. W. (1990). The development of the dizziness handicap inventory. Archives of Otolaryngology - Head and Neck Surgery, 116(4), 424-427. https://doi.org/10.1001/archotol.1990.01870040046011

Jacobson, G. P., Newman, C. W., Hunter, L., \& Balzer, G. K. (1991). Balance function test correlates of the dizziness handicap inventory. Journal of the American Academy of Audiology, 2(4), 253-260.

Jønsson, L. R., Kristensen, M. T., Tibaek, S., Andersen, C. W., \& Juhl, C. (2011). Intra- and interrater reliability and agreement of the Danish version of the Dynamic Gait Index in older people with balance impairments. Archives of Physical Medicine and Rehabilitation, 92(10), 1630-1635. https://doi.org/10.1016/j.apmr.2011.04.020

Kristensen, M. T., Bloch, M. L., Jønsson, L. R., \& Jakobsen, T. L. (2019). Interrater reliability of the standardized Timed Up and Go Test when used in hospitalized and communitydwelling older individuals. Physiotherapy Research International, 24(2), e1769. https://doi.org/10.1002/pri.1769

Loughran, S., Tennant, N., Kishore, A., \& Swan, I. R. (2005). Interobserver reliability in evaluating postural stability between clinicians and posturography. Clinical Otolaryngology, 30(3), 255-257. https://doi.org/10.1111/j.1365-2273.2005.00988.x

Maarsingh, O. R., Stam, H., van de Ven, P. M., van Schoor, N. M., Ridd, M. J., \& van der Wouden, J. C. (2014). Predictors of dizziness in older persons: a 10-year prospective cohort study in the community. BMC Geriatrics, 14, 133. https://doi.org/10.1186/1471-2318-14-133

Mueller, M., Strobl, R., Jahn, K., Linkohr, B., Ladwig, K. H., Mielck, A., \& Grill, E. (2014). Impact of vertigo and dizziness on self-perceived participation and autonomy in older adults: results from the KORA-Age study. Quality of Life Research, 23(8), 2301-2308. https://doi.org/10.1007/s11136-014-0684-x

O'Sullivan, S.B., Schmitz, T.J. \& Fulk, G.D. (2019). Physical Rehabilitation (7th ed.). F.A. Davis.
Podsiadlo, D., \& Richardson, S. (1991). The timed "Up \& Go": a test of basic functional mobility for frail elderly persons. Journal of the American Geriatrics Society, 39(2), 142-148. https://doi.org/10.1111/j.1532-5415.1991.tb01616.x

Portney, L. G., \& Watkins, M. P. (2009). Foundations of Clinical Research: Applications to Practice (3rd ed.). Pearson International Edition.

Shumway-Cook, A., Baldwin, M., Polissar, N. L., \& Gruber, W. (1997). Predicting the probability for falls in communitydwelling older adults. Physical Therapy, 77(8), 812-819. https://doi.org/10.1093/ptj/77.8.812

Shumway-Cook, A., Brauer, S., \& Woollacott, M. (2000). Predicting the probability for falls in community-dwelling older adults using the timed up \& go test. Physical Therapy, 80(9), 896-903. https://doi.org/10.1093/ptj/80.9.896

Vereeck, L., Truijen, S., Wuyts, F. L., \& Van de Heyning, P. H. (2007). The dizziness handicap inventory and its relationship with functional balance performance. Otology \& Neurotology, 28(1), 87-93. https://doi.org/10.1097/01.mao.0000247821.98398.0d

Whitney, S. L., Hudak, M. T., \& Marchetti, G. F. (1999). The activities-specific balance confidence scale and the dizziness handicap inventory: a comparison. Journal of Vestibular Research: Equilibrium \& Orientation, 9(4), 253-259. https://doi.org/10.3233/VES-1999-9402

Whitney, S. L., Marchetti, G. F., Schade, A., \& Wrisley, D. M. (2004). The sensitivity and specificity of the timed "up \& go" and the dynamic gait index for self-reported falls in persons with vestibular disorders. Journal of Vestibular Research : Equilibrium \& Orientation, 14(5), 397-409. https://doi.org/10.3233/VES-2004-14506

Whitney, S. L., \& Wrisley, D. M. (2004). The influence of footwear on timed balance scores of the modified clinical test of sensory interaction and balance. Archives of Physical Medicine and Rehabilitation, 85(3), 439-443. https://doi.org/10.1016/j.apmr.2003.05.005

Whitney, S. L., Wrisley, D. M., Brown, K. E., \& Furman, J. M. (2004). Is perception of handicap related to functional performance in persons with vestibular dysfunction? Otology \& Neurotology, 25(2), 139-143. https://doi.org/10.1097/00129492-200403000-00010

Whitney, S., Wrisley, D., \& Furman, J. (2003). Concurrent validity of the Berg Balance Scale and the Dynamic Gait Index in people with vestibular dysfunction. Physiotherapy Research International, 8(4), 178-186. https://doi.org/10.1002/pri.288

Wrisley, D. M., Walker, M. L., Echternach, J. L., \& Strasnick, B. (2003). Reliability of the dynamic gait index in people 
with vestibular disorders. Archives of Physical Medicine and Rehabilitation, 84(10), 1528-1533.

https://doi.org/10.1016/s0003-9993(03)00274-0 